OPEN ACCESS

Edited by:

Chihaya Adachi,

Kyushu University, Japan

Reviewed by:

Kai Wang,

Soochow University, China Jang Hyuk Kwon,

Kyung Hee University, South Korea Jean Charles Ribierre,

Kyushu University, Japan

*Correspondence:

Hisahiro Sasabe

h-sasabe@yz.yamagata-u.ac.jp

Junji Kido

kid@yz.yamagata-u.ac.jp

Specialty section:

This article was submitted to

Organic Chemistry,

a section of the journal

Frontiers in Chemistry

Received: 27 December 2019

Accepted: 23 April 2020

Published: 25 May 2020

Citation:

Sasabe H, Chikayasu Y, Ohisa S, Arai $H$, Ohsawa T, Komatsu R, Watanabe $Y$, Yokoyama $D$ and Kido J (2020) Molecular Orientations of Delayed Fluorescent Emitters in a Series of Carbazole-Based Host Materials. Front. Chem. 8:427. doi: 10.3389/fchem.2020.00427

\section{Molecular Orientations of Delayed Fluorescent Emitters in a Series of Carbazole-Based Host Materials}

\author{
Hisahiro Sasabe ${ }^{1,2,3 *}$, Yuki Chikayasu ${ }^{3}$, Satoru Ohisa ${ }^{1,2,3}$, Hiroki Arai $^{3}$, Tatsuya Ohsawa ${ }^{3}$, \\ Ryutaro Komatsu ${ }^{3}$, Yuichiro Watanabe ${ }^{3}$, Daisuke Yokoyama ${ }^{1,2,3}$ and Junji Kido ${ }^{1,2,3 *}$ \\ ${ }^{1}$ Research Center for Organic Electronics (ROEL), Yamagata University, Yamagata, Japan, ${ }^{2}$ Frontier Center for Organic \\ Materials (FROM), Yamagata University, Yamagata, Japan, ${ }^{3}$ Department of Organic Materials Science, Graduate School of \\ Organic Materials Science, Yamagata University, Yamagata, Japan
}

Molecular orientation is one of the most crucial factors to boost the efficiency of organic light-emitting devices. However, active control of molecular orientation of the emitter molecule by the host molecule is rarely realized so far, and the underlying mechanism is under discussion. Here, we systematically investigated the molecular orientations of thermally activated delayed fluorescence (TADF) emitters in a series of carbazole-based host materials. Enhanced horizontal orientation of the TADF emitters was achieved. The degree of enhancement observed was dependent on the host material used. Consequently, our results indicate that $\pi-\pi$ stacking, $\mathrm{CH} / \mathrm{n}(\mathrm{n}=\mathrm{O}, \mathrm{N})$ weak hydrogen bonds, and multiple $\mathrm{CH} / \pi$ contacts greatly induce horizontal orientation of the TADF emitters in addition to the molecular shape anisotropy. Finally, we fabricated TADF-based organic light-emitting devices with an external quantum efficiency ( $\eta_{\text {ext }}$ ) of $26 \%$ using an emission layer with horizontal orientation ratio $(\Theta)$ of $79 \%$, which is higher than that of an almost randomly oriented emission layer with $\Theta$ of $62 \%$ ( $\left.\eta_{\text {ext }}=22 \%\right)$.

Keywords: delayed fluorescence, molecular orientation, light out-coupling efficiency, carbazoles, weak hydrogen bonds

\section{INTRODUCTION}

A series of fluorescent emitters exhibiting significant delayed fluorescence, so-called thermally activated delayed fluorescent (TADF) emitters, has attracted much attention due to its potential usefulness in high-performance organic light-emitting devices (OLEDs) that can realize an internal quantum efficiency $\left(\eta_{\text {int }}\right)$ of $100 \%$ (Uoyama et al., 2012; Sasabe and Kido, 2013; Adachi, 2014; Kaji et al., 2015; Lin et al., 2016; Im et al., 2017; Wong and Zysman-Colman, 2017; Yang et al., 2017; Komatsu et al., 2018). In principle, TADF emitters consist of electron-donor (D) and electronacceptor (A) moieties realizing efficient intramolecular charge transfer (ICT). The connection between $\mathrm{D}$ and A moieties is generally accompanied with a small overlap in the frontier molecular orbital (FMO) between the highest occupied molecular orbital (HOMO) and the lowest unoccupied molecular orbital (LUMO), in other words, a small energy difference between singlet and triplet energies $\left(\Delta E_{S T}\right)$. Recent rapid development in TADF emitters enables OLEDs to achieve an external quantum efficiency $\left(\eta_{\text {ext }}\right.$ ) over 30\% (Kaji et al., 2015; Komino et al., 2016; Lin et al., 2016; Liu et al., 2017; Rajamalli et al., 2017; Wu et al., 2018; Ahn et al., 2019; Kondo et al., 2019). To obtain such a high-performance in OLEDs, horizontal orientation of the emission dipole moment (EDM) is absolutely essential. Perfect horizontal orientation of the EDM has been reported to boost OLED 
efficiency up to $150 \%$ compared to random EDM orientation (Frischeisen et al., 2011; Yokoyama, 2011; Schmidt et al., 2017; Kim and Kim, 2018; Watanabe et al., 2019b). However, the horizontal orientation ratio $(\Theta)$ of most TADF emitters used in OLEDs realizing $\eta_{\text {ext }}$ over $30 \%$ is reportedly only around $80 \%$, which is far behind perfect horizontal orientation $(\Theta=$ $100 \%)$. In general, a guest TADF emitter is dispersed into a host material to reduce concentration quenching, and to maintain carrier balance in the emission layer (EML). The proportion of guest molecule is commonly much smaller than that of host molecule. Thus, the non-covalent interactions between host and guest molecules should be one of the important factors that controls the molecular orientation.

In an early stage of study on molecular orientation in OLEDs, Yokoyama used variable-angle spectroscopic ellipsometry (VASE) measurements to reveal the anisotropy of the molecular shape, such as in planar and linear structures, is essential to realize horizontal orientation of a series of triphenylamine and carbazole based-fluorescent molecules, even in randomly oriented host molecules (Yokoyama et al., 2009). However, unlike first-generation fluorescent molecules, most TADF molecules do not have a planar structure but instead a winding chemical structure in needed to realize a small overlap of the frontier molecular orbitals in order to achieve efficient reverse intersystem crossing (RISC). Therefore, in addition to the molecular shape anisotropy, advanced strategies to actively use non-covalent interactions to realize enhanced horizontal orientation are highly desired.

In 2016, Lin and Wong reported three types of skyblue TADF emitters using triphenyltriazine as an acceptor and substituted acridines as a donor unit (Lin et al., 2016). Among these, SpiroAc-TRZ with spirobiphenyl unit showed a photoluminescent quantum yield ( $\eta_{\mathrm{PL}}$ ) of $100 \%$, and a high $\Theta$ value of $83 \%$ in $\mathbf{m C P C N}$ host. They indicated that this high $\Theta$ value of SpiroAc-TRZ is attributed to the overall planar and balanced/symmetrical structure. The corresponding OLED exhibited an extremely high $\eta_{\text {ext }}$ of nearly $37 \%$. In the same year, Komino and Adachi reported the complete horizontal orientation of a linear-shaped TADF emitter named Cis-BOX2 in a randomly oriented host matrix at the temperature of $200 \mathrm{~K}$ (Komino et al., 2016). The horizontal orientation ratio of CisBOX2 depended on the deposition temperature and the type of host matrix. A Cis-BOX2-based OLED showed a very high $\eta_{\text {ext }}$ of $33 \%$.

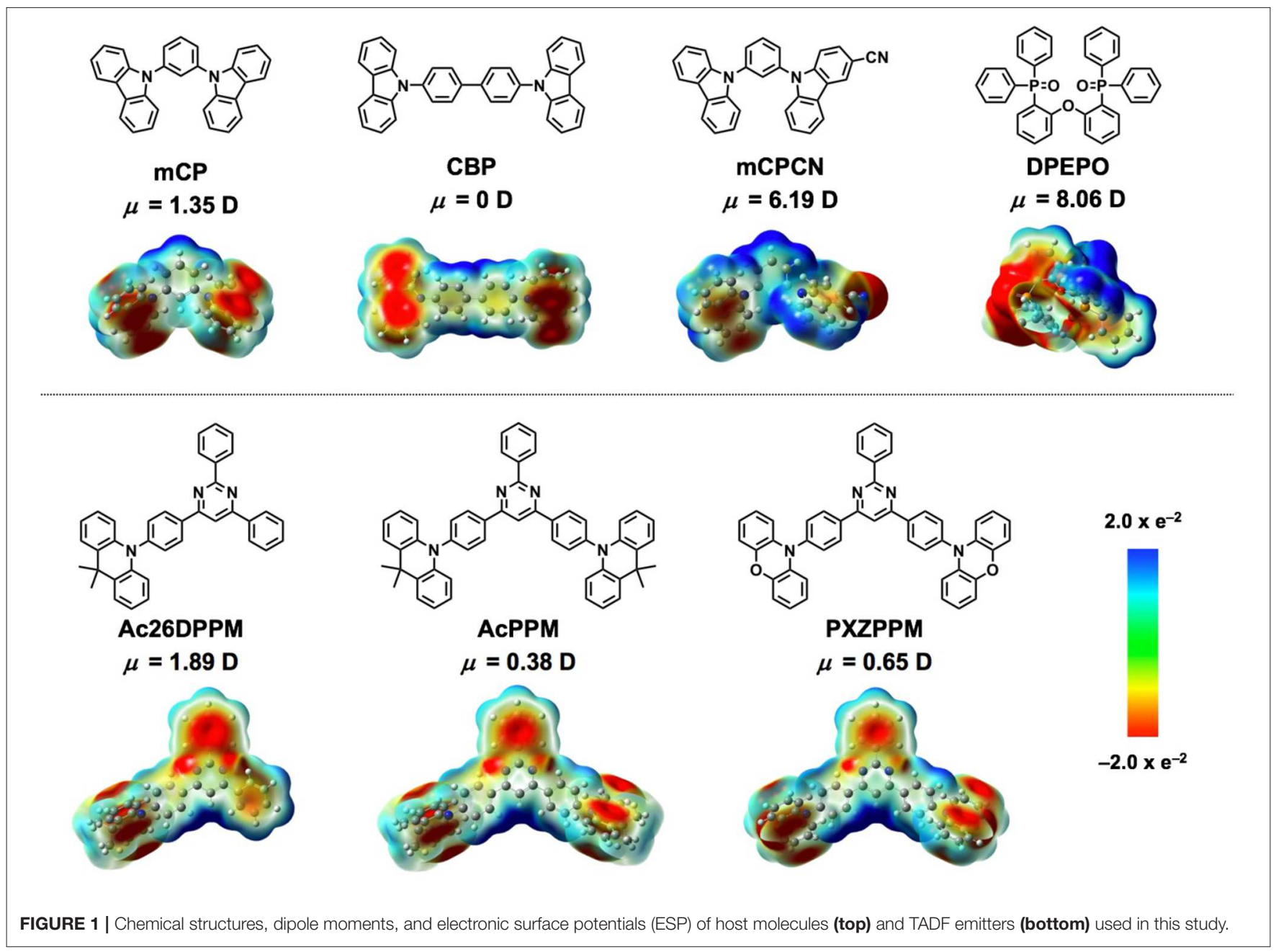


As mentioned above, although a high $\Theta$ value of up to $100 \%$ at $200 \mathrm{~K}$ has been reported, the underlying mechanism to realize horizontal orientation is still under discussion at this stage (Yokoyama, 2011; Mayr and Brütting, 2015; Moon et al., 2015; Shibata et al., 2015; Friederich et al., 2017; Schmidt et al., 2017; Gujral et al., 2018; Kim and Kim, 2018; Lee et al., 2018; Pal et al., 2018; Watanabe et al., 2019b). In order to deepen insights into the underlying mechanism, we wish to report a systematic investigation on the molecular orientations of TADF molecules in a series of carbazole-based host materials. To reveal the structure-property relationship for horizontal orientation, a series of TADF emitters, which we can systematically change the parameters one by one, should be necessary. In this context, we have already developed a series of pyrimidine-based TADF emitters with different donor group(s) (Figure 1) (Komatsu et al., 2016a,b; Nakao et al., 2017). By comparing these three molecules, we can obtain insight into the effects of two factors: (i) donor number, which reflects the molecular shape anisotropy in these comparisons, and (ii) donor structure. Consequently, we achieved enhanced $\Theta$ values of TADF emitters and the degree of enhancement depended on the host material used. Consequently, our results indicate that increased $\pi-\pi$ stacking, $\mathrm{CH} / \mathrm{n}(\mathrm{n}=\mathrm{O}, \mathrm{N})$ weak hydrogen bond (H-bond) interaction, and multiple $\mathrm{CH} / \pi$ contacts greatly induce horizontal orientation of TADF emitters in addition to the molecular shape anisotropy. Finally, we fabricated a TADFbased OLED with $\eta_{\text {ext }}$ of $26 \%$ using an emission layer (EML) with a $\Theta$ value of $79 \%$, which is greater than that of an almost randomly oriented EML with a $\Theta$ value of $62 \%\left(\eta_{\text {ext }}\right.$ $=22 \%)$.

\section{RESULTS AND DISCUSSION}

\section{Selection of Carbazole-Based Host Molecules and Phosphine-Oxide Host Molecules}

Carbazole derivatives are one of the most popular host materials in OLEDs. Therefore, insights into carbazole-based host materials are considered to be highly valuable. To identify the underlying mechanisms of horizontal orientation, we selected four types of host materials to study and sequentially changed their chemical structures (Figure 1 and Table 1). mCP was used as the benchmark material. Extending its $\pi$-conjugation, results in CBP, which can be used to evaluate the effect of $\pi$-conjugation or $\pi-\pi$ stacking when compared to $\mathbf{m C P}$. The introduction of $\mathrm{CN}$ substituent into $\mathbf{m C P}$ forms $\mathbf{m C P C N}$ (Lin et al., 2012). Using this molecule, we can validate the effect of $\mathrm{CN}$ substituent, such as the dipole/dipole interaction and $\mathrm{CH} / \mathrm{N}$ weak H-bonds. A phosphine-oxide host material, DPEPO (Han et al., 2011) was used to investigate the effect of the dipole moment and $\mathrm{CH} / \mathrm{O}$ weak $\mathrm{H}$-bonds of $\mathrm{P}=\mathrm{O}$ substituents (Figure 2). Note that all the host materials used in this study showed random orientation. DFT calculations were performed at B3LYP 6-31G(d) level to evaluate the dipole moment and visualize the electronic surface potential (ESP), as shown in
TABLE 1 | Thermal and optical properties of the host molecules.

\begin{tabular}{lcccc}
\hline Compound & $\boldsymbol{M w}(\mathbf{g} / \mathbf{m o l})$ & $\left.\boldsymbol{T}_{\boldsymbol{g}}^{\mathbf{a}} / \boldsymbol{T}_{\boldsymbol{m}}^{\mathbf{a}} / \boldsymbol{T}_{\boldsymbol{d} \mathbf{b}}^{\boldsymbol{b}}{ }^{\circ} \mathbf{C}\right)$ & $\boldsymbol{I}_{\boldsymbol{p}}^{\mathbf{e}} / \boldsymbol{E}_{\mathbf{g}}{ }^{\boldsymbol{f}} / \boldsymbol{E}_{\mathrm{a}}{ }^{g} / \boldsymbol{E}_{\mathrm{T}}{ }^{\boldsymbol{h}}(\mathbf{e V})$ & $\boldsymbol{S}^{\boldsymbol{i}}$ \\
\hline $\mathrm{mCP}$ & 408 & $60 /$ n.d. $/ 280$ & $-6.01 / 3.49 /-2.63 / 3.00$ & 0.08 \\
$\mathrm{CBP}$ & 485 & $62 / 283 / 413$ & $-5.91 / 3.44 /-2.67 / 2.60$ & -0.07 \\
$\mathrm{mCPCN}$ & 434 & $97 / 222 / 313$ & $-6.08 / 3.44 /-2.64 / 3.03$ & 0.08 \\
DPEPO & 571 & n.d./280/322 & $-6.70 / 4.00 /-2.70 / 3.30$ & 0.00 \\
\hline
\end{tabular}

${ }^{a} T_{g}$ and $T_{m}$ were determined using DSC. ${ }^{b} T_{d 5}$ was determined using TGA. ${ }^{e} / p$ was determined using PYS. ${ }^{f} E_{g}$ was taken as the point where the normalized absorption spectra intersected. ${ }^{g} E_{a}$ was calculated using $I_{p}$ and $E_{g} .{ }^{h} E_{T}$ was estimated from the onset of the phosphorescent spectra at $5 \mathrm{~K}$. 'VASE derived order parameter.

TABLE 2 | Thermal and optical properties of the TADF emitters.

\begin{tabular}{lccc}
\hline Compound & Mw (g/mol) & $\left.\boldsymbol{T}_{\boldsymbol{g}}^{a} / \boldsymbol{T}_{\boldsymbol{m}}^{a} / \boldsymbol{T}_{\boldsymbol{d} \mathbf{b}}^{b}{ }^{\circ} \mathbf{C}\right)$ & $\boldsymbol{I}_{\boldsymbol{p}}^{\mathbf{e}} / \boldsymbol{E}_{\mathrm{g}}{ }^{\boldsymbol{}} / \boldsymbol{E}_{\mathrm{a}}{ }^{g} / \boldsymbol{E}_{\mathrm{T}}{ }^{\boldsymbol{h}}(\mathbf{e V})$ \\
\hline Ac-26DPPM & 516 & $90 / 210 / 383$ & $-5.67 / 2.90 /-2.77 / 2.80$ \\
Ac-PPM & 723 & n.d./388/442 & $-5.65 / 2.80 /-2.85 / 2.65$ \\
PXZ-PPM & 671 & n.d./290/473 & $-5.65 / 2.56 /-3.09 / 2.56$ \\
\hline
\end{tabular}

${ }^{a} T_{g}$ and $T_{m}$ were determined using DSC. ${ }^{b} T_{d 5}$ was determined using TGA. ${ }^{e} I_{p}$ was determined using PYS. ${ }^{f} E_{g}$ was taken as the point where the normalized absorption spectra intersected. ${ }^{g} E_{a}$ was calculated using $I_{p}$ and $E_{g} .{ }^{h} E_{T}$ was estimated from the onset of the phosphorescent spectra at $5 \mathrm{~K}$.

Figure 1 and Figure S1 (in supporting information). Among the host materials, $\mathbf{m C P}$ and $\mathbf{C B P}$ had small dipole moment $<1.5 \mathrm{D}$, while $\mathbf{m C P C N}$ with a cyano group and DPEPO with phosphine oxide groups possessed a large dipole moment $>6.0$ D. ESP exhibited strong negative charge (red color) on the $\pi$-plane of the carbazole and on the electron-withdrawing groups of $\mathrm{CN}$ and $\mathrm{P}=\mathrm{O}$. The hydrogen $(\mathrm{H})$ atoms on the aromatic rings had positive charge (blue color).

\section{Selection of TADF Emitters}

We selected three types of pyrimidine-based TADF emitters named Ac26DPPM (Nakao et al., 2017), AcPPM (Komatsu et al., 2016a), and PXZPPM (Komatsu et al., 2016b) with different donor group(s) such as dimethylacridine (Ac) and phenoxiazine (PXZ) (Figure 1 and Table 2). By comparing these three molecules, we can obtain insight into the effects of two factors: (i) donor number, which reflects the molecular shape anisotropy in these comparisons, and (ii) donor structure. Note that all the emitter molecules showed random orientation in neat film (Figure S2). Similar to the host molecules, DFT calculations were performed to evaluate the dipole moment and visualize the ESP and the results are shown in Figure 1 and Figure S1. All the emitters have a small dipole moment $<2.0 \mathrm{D}$, especially AcPPM $(\mu=0.38 \mathrm{D})$ and PXZPPM $(\mu=0.65 \mathrm{D})$. The ESP indicates strong negative charge on the $\pi$-plane of position 2 of pyrimidine and on the electron-donating end-cap unit(s) of Ac and PXZ. Similar to the host molecules, $\mathrm{H}$ atoms on the aromatic rings had positive charge. 


\section{Molecular Orientation of TADF Emitters}

First, we investigated the molecular orientations of $10 \mathrm{wt} \%$ Ac26DPPM-doped host films by using angle dependent PL measurements. Here, a $\Theta$ value of $67 \%$ indicates random orientation while a value of $100 \%$ indicates perfect horizontal orientation. Ac26DPPM-doped mCP film showed moderate vertical orientation with $\Theta=53 \%$. When Ac26DPPM was doped into other host materials (CBP, mCPCN, and DPEPO), all the doped films exhibited almost random orientation with $\Theta$ of $62-66 \%$ (Figure 3 and Figure S3). Although these films showed random orientation due to the small anisotropy of Ac26DPPM, the differences of orientation ratio in the different host materials $(\Delta \Theta)$ were $9-13 \%$. These differences are not small but meaningfully significant. Similar results were obtained in the case of AcPPM (Figure 4 and Figure S4). When host material was changed from $\mathbf{m C P}$ to one of the other host materials, a significant enhancement of $\Theta$ values up to $22 \%$ was observed. Since AcPPM has larger molecular shape anisotropy than that of Ac26PMM, AcPPM showed increased horizontal orientation with $\Theta$ of $75-80 \%$. Surprisingly, PXZPPM also exhibited similar tendencies as the Ac-end-capped emitters (Figure 5 and Figure S5). When $\mathbf{m C P}$ was used, the PXZPPM/mCP film showed almost random orientation with $\Theta$ of $62 \%$, while when PXZPPM was doped into the other host materials, all the doped films exhibited significant horizontal orientation with $\Theta$ of $74-81 \%$. Among these emitters, it was determined that bulky methyl groups on Ac end-capping groups do not cause a negative effect toward horizontal orientation as the emitter without methyl groups showed very similar $\Theta$ values.

\section{Underlying Mechanism for Horizontal Orientation}

To validate the underlying mechanisms for horizontal orientation, we compared the chemical structures of the host materials. When host was changed from $\mathbf{m C P}$ to $\mathbf{C B P}$, $\pi$-conjugation is expanded from phenyl to a biphenyl linker. Therefore, enhanced $\pi-\pi$ stacking can be considered as an additional interaction. Note that the dipole moment of CBP is calculated to be $0 \mathrm{D}$, while that of $\mathbf{m C P}$ is $1.35 \mathrm{D}$. Further, the dipole moments of the emitters are smaller than $2.0 \mathrm{D}$. Thus, dipole/dipole interaction can be ruled out as a major interaction. In the case of $\mathbf{m C P C N}$, the difference of chemical structure is only a $\mathrm{CN}$ group on the carbazole plane. Since the $\mathrm{CN}$ group is a strong electron-withdrawing group, the resulting $\mathbf{m C P C N}$ has a large dipole moment of 6.19 D. However, as mentioned in the case of CBP, the contributions from dipole/dipole interaction can be considered to be small because the emitters used in this study have very small dipole moment $<2 \mathrm{D}$. In fact, the $\Delta \Theta$ of Ac26DPPM $(\Delta \Theta=9 \%)$ with a larger dipole moment ( $\mu$ $=1.89 \mathrm{D})$ was smaller than that of AcPPM $(\Delta \Theta=17 \%)$ with a smaller dipole moment $(\mu=0.38 \mathrm{D})$. Therefore, it is only $\mathrm{CH} / \mathrm{N}$ weak $\mathrm{H}$-bond of $\mathrm{CN}$ group plays a key role. The binding energy of $\mathrm{CH} / \mathrm{N}$ weak $\mathrm{H}$-bond is $10-20 \mathrm{~kJ} / \mathrm{mol}$ (Desiraju and Steiner, 1999). This interaction has been reported to be a key to controlling horizontal orientation of oligopyridine-containing electron transporters (Sasabe et al., 2011; Yokoyama et al., 2011; Watanabe et al., 2019a,b). DPEPO is a frequently used host material in high efficiency TADF OLEDs, and the films using it as the host showed the largest $\Theta$ values among the four host materials. DPEPO has a large dipole moment of $8.06 \mathrm{D}$, and therefore the contributions from dipole/dipole interaction are considered to be large. However, similar to the case of $\mathbf{m C P C N}$, the contributions from dipole/dipole interaction can be considered to be small because the emitters used in this study have very small dipole moment $<2 \mathrm{D}$. In fact, the $\Delta \Theta$ of Ac26DPPM $(\Delta \Theta=13 \%)$ with a larger dipole moment $(\mu=1.89 \mathrm{D})$ was smaller than that of AcPPM $(\Delta \Theta=$ $22 \%)$ with a smaller dipole moment $(\mu=0.38 \mathrm{D})$. Given that DPEPO has short conjugation lengths with steric hindrance, $\pi-\pi$ interaction is relatively small. Thus, it can be considered that $\mathrm{CH} / \mathrm{O}$ weak $\mathrm{H}$-bond from $\mathrm{P}=\mathrm{O}$ and ether linker plays a key role in determining the horizontal orientation when DPEPO is used as the host material. Recently, Samuel and Zysman-Colman proposed that the significant number of electronegative sites from $\mathrm{P}=\mathrm{O}$ and ether linker on the surface of DPEPO vacuumdeposited film plays an important role for horizontal molecular orientation (Pal et al., 2018). Further, it has also been reported that the strong acceptor property of $\mathrm{P}=\mathrm{O}$ forms shorter $\mathrm{CH} \cdots \mathrm{O}$ $\mathrm{H}$-bond, and the bond length of the $\mathrm{CH} \cdots \mathrm{O} \mathrm{H}$-bond is relatively shorter than that of $\mathrm{CH} \cdots \mathrm{N} \mathrm{H}$-bond (Desiraju and Steiner, 1999).

Among the three emitters, PXZPPM and AcPPM showed similar tendencies for horizontal orientation, even though AcPPM has four bulky methyl groups on the acridine end-capping units. One possible reason for this is the contributions from multiple $\mathrm{CH} / \pi$ contacts between the methyl groups and the $\pi$ planes. $\mathrm{CH} / \pi$ contact is a very small interaction, and the binding energy is only $2-3 \mathrm{~kJ} / \mathrm{mol}$ (Nishio et al., 1998). However, when the molecule has a large number of methyl groups, the contributions would not be negligible in the solid state. In fact, very recently, enhanced horizontal orientation is realized in a series of iridium complexes by introduction of methyl or alkyl groups (Shin et al., 2019). Therefore, we propose that the contributions from multiple $\mathrm{CH} / \pi$ contacts can collaborate with other strong intermolecular interactions to realize significant horizontal orientation.

\section{OLED Performances}

Finally, we fabricated OLEDs using PXZPPM/hosts as an emission layer to show the effect of horizontal orientation on the OLED efficiency. Here, we used two types of hosts, mCP $(\Theta=62 \%)$ and $\mathbf{m C P C N}(\Theta=79 \%)$. Note that we did not use DPEPO as a host material because the chemical structure is totally different from $\mathrm{mCP}$. The structure of the OLED was [indium tin oxide (ITO) anode $(130 \mathrm{~nm}) /$ triphenylaminecontaining polymer: 4-isopropyl-4'-methyldiphenyliodonium tetrakis(pentafluorophenyl)borate (PPBI) (20 nm) (Kido et al., 1997)/di-[4-(N,N-ditolyl-amino)-phenyl]cyclohexane (TAPC) $\quad(25 \mathrm{~nm}) / 4,4^{\prime}, 4^{\prime \prime}$-tris(N-carbazolyl)triphenylamine (TCTA) $(5 \mathrm{~nm}) / 10 \mathrm{wt} \%$ PXZPPM-doped $\mathbf{m C P}$, or $\mathbf{m C P C N}$ $(10 \mathrm{~nm}) / 3,3^{\prime \prime}, 5,5^{\prime}$-tetra(3-pyridyl)-1,1'; $3^{\prime}, 1^{\prime \prime}$-terphenyl (B3PyPB) 

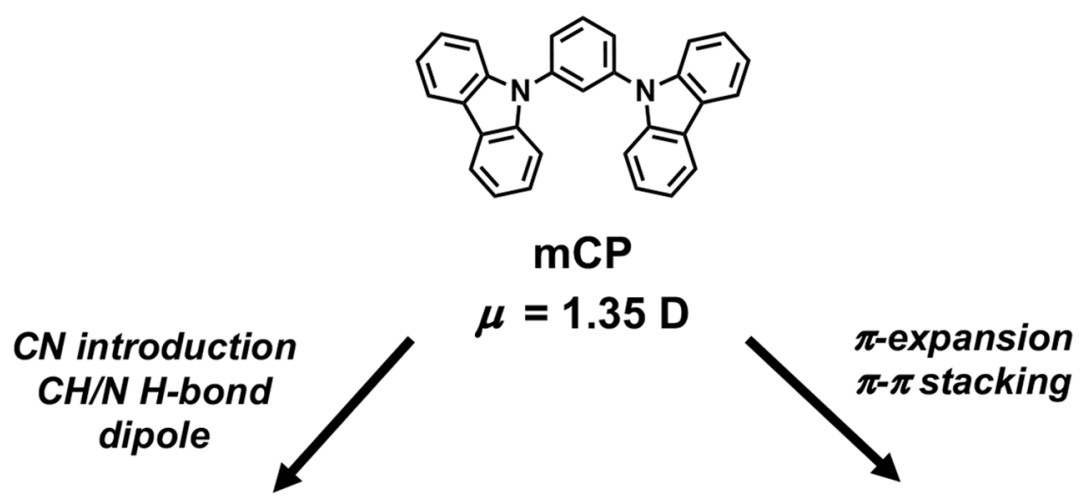

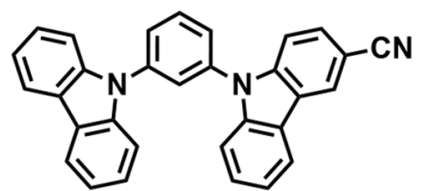

mCPCN $\mu=6.19 \mathrm{D}$<smiles>c1ccc2c(c1)c1ccccc1n2-c1ccc(-c2ccc(-n3c4ccccc4c4ccccc43)cc2)cc1</smiles>

\section{CBP \\ $\mu=0 \mathrm{D}$}<smiles>O=P(c1ccccc1)(c1ccccc1)c1ccccc1Oc1ccccc1P(=O)(c1ccccc1)c1ccccc1</smiles>

$\mu=8.06 \mathrm{D}$

$P=O$, ether linker $\mathrm{CH} / \mathrm{O}$ H-bond, dipole

FIGURE 2 | Intermolecular interactions introduced when comparing the host materials to the mCP benchmark.

A

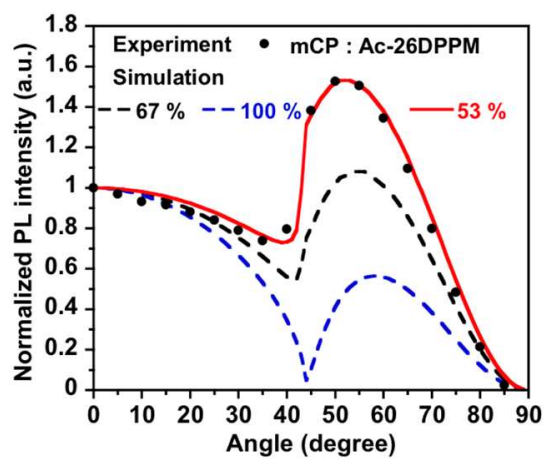

C

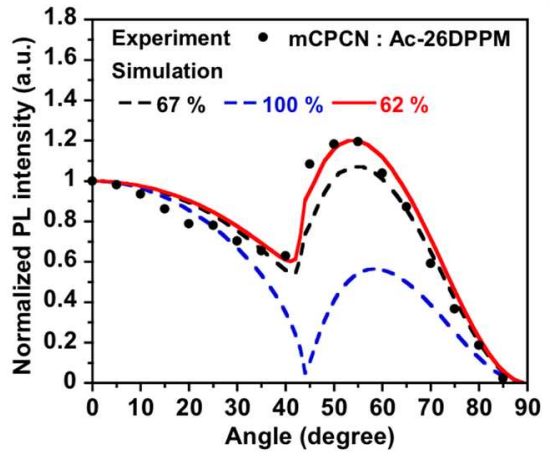

B
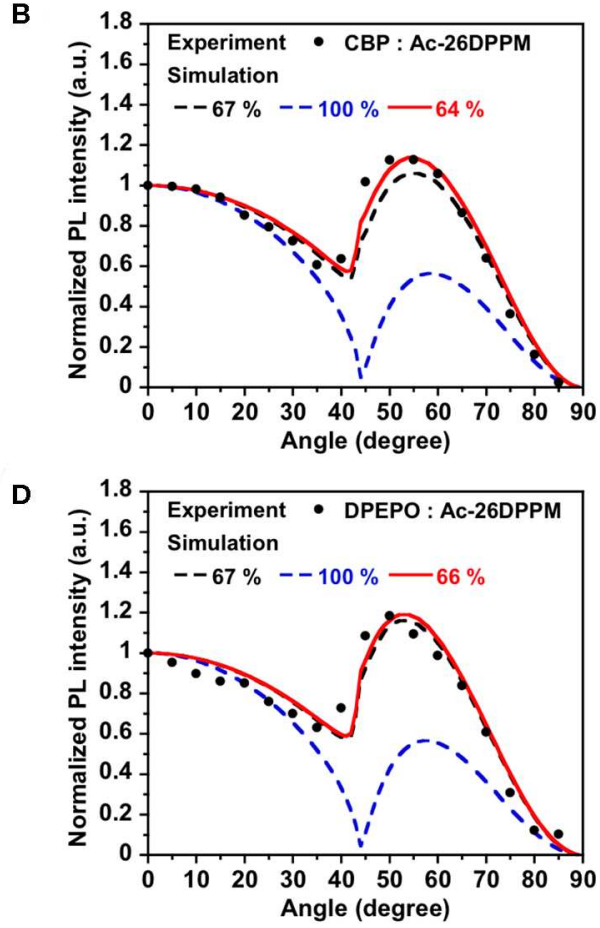

FIGURE 3 | PL intensity of Ac26DPPM-doped host films at different angles. The experimental data are in comparison with the fitting curve for different horizontal dipole ratios for Ac26DPPM doped in a host film of (A) mCP, (B) CBP, (C) mCPCN, and (D) DPEPO. 
A

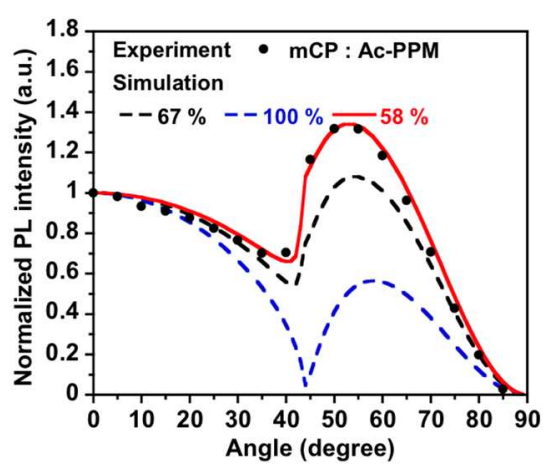

C

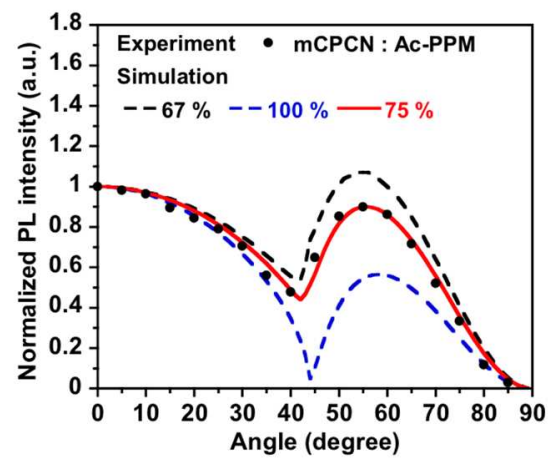

B

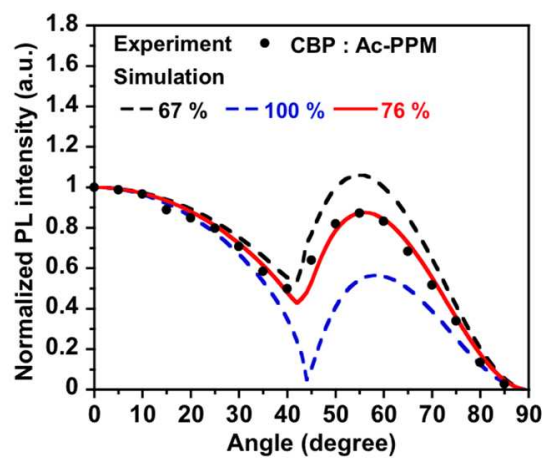

D

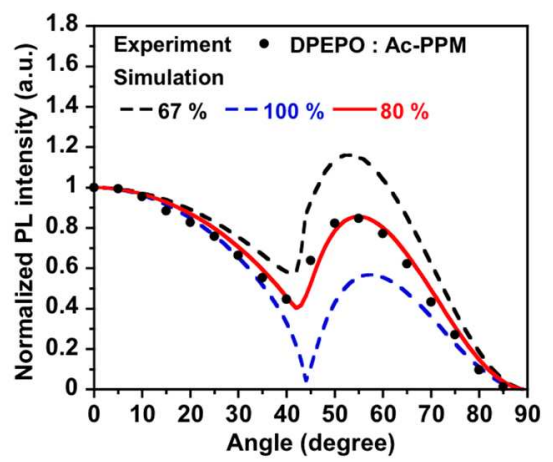

FIGURE 4 | PL intensity of AcPPM-doped in (A) mCP, (B) CBP, (C) $\mathbf{m C P C N}$, and (D) DPEPO host films at different angles. The experimental data are in comparison with the fitting curve for different horizontal dipole ratios.

A

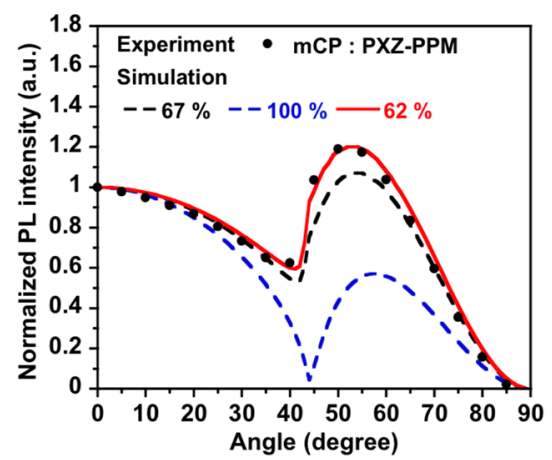

C

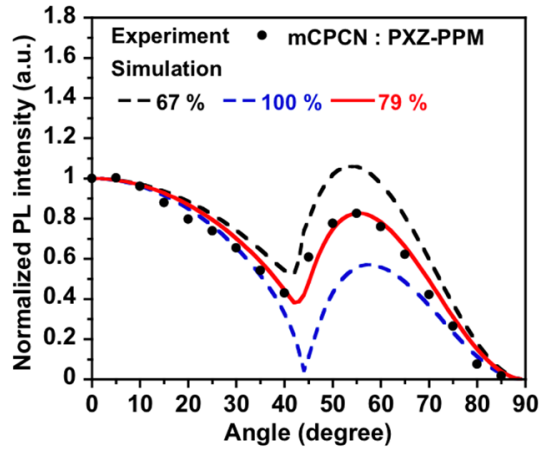

B

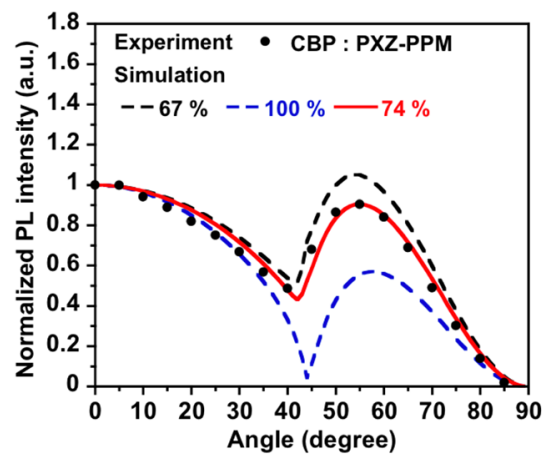

D

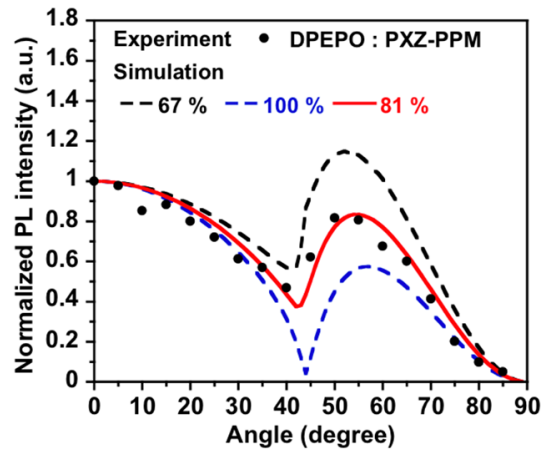

FIGURE 5 | PL intensity of PXZPPM-doped in (A) MCP, (B) CBP, (C) $\mathbf{m C P C N}$, and (D) DPEPO host films at different angles. The experimental data are in comparison with the fitting curve for different horizontal dipole ratios. 
A

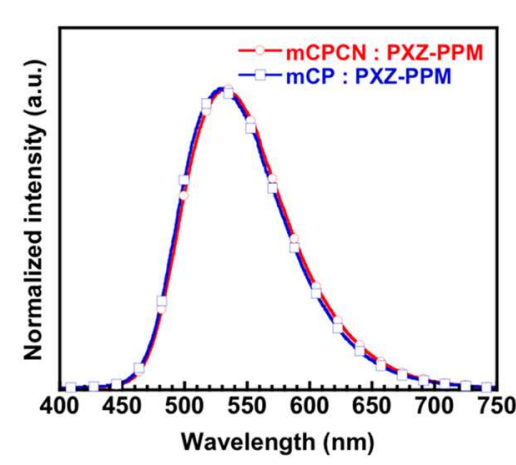

C

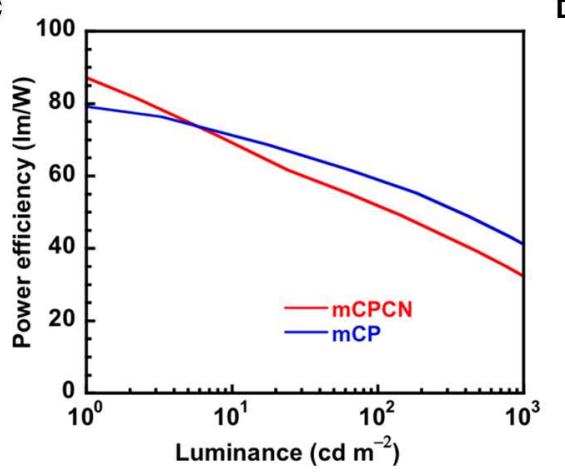

B

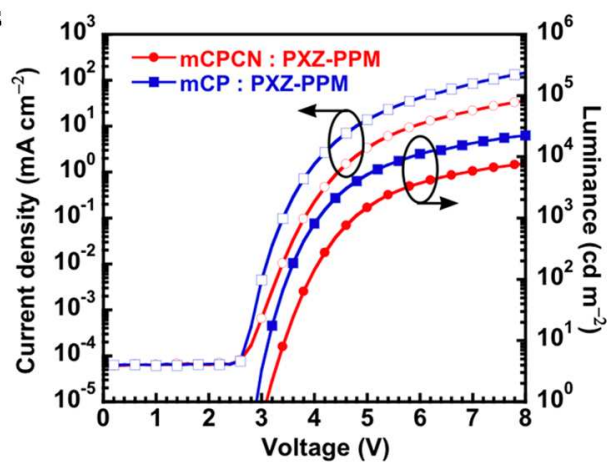

D

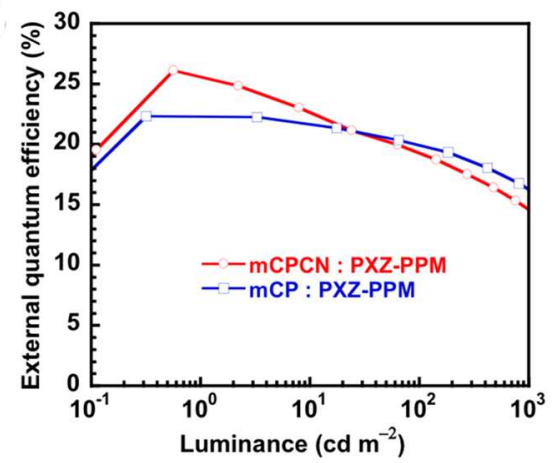

FIGURE 6 | Device performance of PXZPPM-based OLEDs; (A) EL spectra; (B) current density-voltage-luminance characteristics; (C) power efficiency-luminance characteristics; (D) external quantum efficiency-luminance characteristics.

TABLE 3 | Summary of PXZPPM-based OLED performance.

\begin{tabular}{|c|c|c|c|c|}
\hline Host & $\begin{array}{l}\mathrm{V}_{\mathrm{on}}{ }^{a} \\
(\mathrm{~V})\end{array}$ & $\begin{array}{c}\mathrm{V}_{100} / \eta_{\mathrm{c}, 100} / \eta_{\mathrm{p}, 100} / \eta_{\mathrm{ext}, 100^{b}} \\
\left(\mathrm{~V} / \mathrm{cd} \mathrm{A}^{-1} / \mathrm{lm} \mathrm{W} \mathbf{W}^{-1} / \%\right)\end{array}$ & $\begin{array}{c}\mathbf{V}_{1000} / \eta_{\mathrm{c}, 1000} / \eta_{\mathrm{p}, 1000} / \eta_{\text {ext, } 1000^{c}} \\
\left(\mathrm{~V} / \mathrm{cd} \mathrm{A}^{-1} / \mathrm{Im} \mathrm{W}^{-1} / \%\right)\end{array}$ & $\begin{array}{c}\eta_{\mathrm{c}, \max } / \eta_{\mathrm{p}, \max } / \eta_{\mathrm{ext}, \max }{ }^{d} \\
\left(\mathbf{c d ~ A} \mathbf{A}^{-1} / \mathrm{Im} \mathrm{W} \mathbf{W}^{-1} / \%\right)\end{array}$ \\
\hline $\mathrm{mCP}$ & 2.85 & 3.46/65.7/59.7/20.0 & 4.07/53.5/41.4/16.3 & 73.1/82.1/22.3 \\
\hline
\end{tabular}

${ }^{a}$ Turn-on voltage at $1 \mathrm{~cd} \mathrm{~m} \mathrm{~m}^{-2} .{ }^{b}$ Voltage $\mathrm{N}$ ), current efficiency $\left(\eta_{c}\right)$, power efficiency $\left(\eta_{p}\right)$, and external quantum efficiency $\left(\eta_{\text {ext }}\right)$ at $100 \mathrm{~cd} \mathrm{~m}^{-2},{ }^{c} \mathrm{~V}, \eta_{c}, \eta_{p}$, and $\eta_{\mathrm{ext}}$ at $1,000 \mathrm{~cd} \mathrm{~m}^{-2}$. ${ }^{d} \eta_{c}$, $\eta_{p}$, and $\eta_{\text {ext }}$ at maximum.

$(50 \mathrm{~nm})$ (Sasabe et al., 2008)/LiF $(0.5 \mathrm{~nm}) / \mathrm{Al}$ cathode $(100 \mathrm{~nm})]$. TAPC and TCTA were used as the hole transport layers, B3PyPB as the electron transport layer, and $\mathrm{LiF}$ as the electron injection layer. The chemical structures of these materials are shown in Figure S6. Figure 6 shows the electroluminescence (EL) spectra, the current density (J)-voltage (V)-luminance (L), and $\eta_{\text {ext }}-\mathrm{L}$ characteristics. The electroluminescent characteristics are summarized in Table 3. The EL spectra of both devices showed similar emission with peaks at $529 \mathrm{~nm}$ for $\mathbf{m C P}$ and at $531 \mathrm{~nm}$ for $\mathbf{m C P C N}$ host materials. The maximum $\eta_{\text {ext }}$ values were recorded to be $22.3 \%$ for $\mathbf{m C P}$ and $26.1 \%$ for mCPCN. Moreover, the light distribution patterns evaluated by Lambertian factor of these devices were similar, and recorded to be 0.99 for $\mathbf{m C P}$, and 0.97 for $\mathbf{m C P C N}$, as shown in Figure S6. The photoluminescent quantum yields ( $\eta_{\mathrm{PL}}$ ) of PXZPPM/host films were similar for mCP (71\%) and mCPCN (69\%) hosts. Given that the carrier balance factor is similar at the peak efficiency, the differences in the maximum $\eta_{\text {ext }}$ values can be attributed to the differences in $\Theta$ values of the different host materials. Note that we also fabricated the devices using CBP and DPEPO as a host material. As a result, higher $\eta_{\text {PL }}$ and $\Theta$ values gave superior device performances (Figure S7 and Table S1). Enhancing the horizontal orientation of the emitter apparently increases the light coupling efficiency, thus resulting in a higher $\eta_{\text {ext }}$ value.

\section{CONCLUSION}

We performed a systematic investigation on the molecular orientations of TADF emitters in a series of carbazole-based host materials. TADF emitters used in this study have a small dipole moment $<2 \mathrm{D}$. To validate the underlying mechanisms for horizontal orientation, we changed the chemical structure of $\mathbf{m C P}$ by: (i) extending the $\pi$-conjugation to form $\mathbf{C B P}$, and (ii) the introduction of a $\mathrm{CN}$ substituent to form $\mathrm{mCPCN}$. In addition, a phosphine-oxide host material, DPEPO, was used. Although all the emitters and the host materials had random orientation as a neat film, the emitters with larger molecular 
shape anisotropy, AcPPM and PXZPPM, showed enhanced horizontal orientation up to $\Theta \sim 81 \%$ in all host films except mCP. The third emitter, Ac26DPPM, has a smaller molecular shape anisotropy, and subsequently showed vertical orientation in the $\mathbf{m C P}$ host film $(\Theta=53 \%)$. However, when doped into the other host material, Ac26DPPM showed random orientation $(\Theta \sim 66 \%)$. From these results, it can be considered that (i) an increase in the $\pi$-conjugation leads to stronger $\pi-\pi$ stacking, (ii) the introduction of $\mathrm{CN}$ group induces $\mathrm{CH} / \mathrm{N}$ weak $\mathrm{H}$-bonds, and (iii) the introduction of a strong acceptor $\mathrm{P}=\mathrm{O}$ and ether groups induces $\mathrm{CH} / \mathrm{O} \mathrm{H}$-bonds, and all these interactions enhance the horizontal orientation of TADF emitters. In addition, we propose that the contributions from multiple $\mathrm{CH} / \pi$ contacts can collaborate with other strong intermolecular interactions such as $\pi-\pi$ stacking, $\mathrm{CH} / \mathrm{N}$ and $\mathrm{CH} / \mathrm{O} \mathrm{H}$-bonds to realize significant horizontal orientation. Finally, we fabricated an PXZPPM-based OLED that achieved an $\eta_{\text {ext }}$ of $26 \%$ using an EML with $\Theta$ of $79 \%$, which is higher than that of an almost randomly oriented EML with $\Theta$ of $62 \%\left(\eta_{\text {ext }}=22 \%\right)$. We believe that our results will be beneficial in revealing the underlying mechanism for horizontal orientation leading to superior OLED performances.

\section{DATA AVAILABILITY STATEMENT}

All datasets generated for this study are included in the article/Supplementary Material.

\section{REFERENCES}

Adachi, C. (2014). Third-generation organic electroluminescence materials. Jpn. J. Appl. Phys. 53:60101. doi: 10.7567/JJAP.53.060101

Ahn, D. H., Kim, S. W., Lee, H., Ko, I. J., Karthik, D., Lee, J. Y., et al. (2019). Highly efficient blue thermally activated delayed fluorescence emitters based on symmetrical and rigid oxygen-bridged boron acceptors. Nat. Photonics 13, 540-546. doi: 10.1038/s41566-019-0415-5

Desiraju, G. R., and Steiner, T. (1999). The Weak Hydrogen Bond-In Structural Chemistry and Biology. New York, NY: Oxford University Press.

Friederich, P., Coehoorn, R., and Wenzel, W. (2017). Molecular origin of the anisotropic dye orientation in emissive layers of organic light emitting diodes. Chem. Mater. 29:9528. doi: 10.1021/acs.chemmater.7b03742

Frischeisen, J., Yokoyama, D., Endo, A., Adachi, C., and Brutting, W. (2011). Increased light outcoupling efficiency in dye-doped small molecule organic light-emitting diodes with horizontally oriented emitters. Org. Electron. 12, 809-817. doi: 10.1016/j.orgel.2011.02.005

Gujral, A., Yu, L., and Ediger, M. D. (2018). Anisotropic organic glasses. Curr. Opin. Solid State Mater. Sci. 22, 49-57. doi: 10.1016/j.cossms.2017.11.001

Han, C. M., Zhao, Y. B., Xu, H., Chen, J. S., Deng, Z. P., Ma, D. G., et al. (2011). A simple phosphine-oxide host with a multi-insulating structure: high triplet energy level for efficient blue electrophosphorescence. Chem. Eur. J. 17, 5800-5803. doi: 10.1002/chem.201100254

Im, Y., Kim, M., Cho, Y. J., Seo, J. A., Yook, K. S., and Lee, J. Y. (2017). Molecular design strategy of organic thermally activated delayed fluorescence emitters. Chem. Mater. 29, 1946-1963. doi: 10.1021/acs.chemmater.6b05324

Kaji, H., Suzuki, H., Fukushima, T., Shizu, K., Suzuki, K., Kubo, S., et al. (2015). Purely organic electroluminescent material realizing $100 \%$ conversion from electricity to light. Nat. Commun. 6:8476. doi: 10.1038/ncomms9476

Kido, J., Harada, G., Komada, M., Shionoya, H., and Nagai, K. (1997). Aromaticamine-containing polymers for organic electroluminescent devices. ACS Symposium Ser. 672, 381-394. doi: 10.1021/bk-1997-0672.ch025

\section{AUTHOR CONTRIBUTIONS}

HS and SO conceived the project. HS, YC, SO, and DY interpreted the data and prepared the manuscript and supplementary materials. HS, SO, and JK supervised the project. YC, SO, and DY designed experiments. HA, TO, $\mathrm{RK}$, and YW synthesized and characterized materials. YC, YW, and DY prepared samples, performed variable-angle spectroscopic ellipsometry measurements, and related data analysis. YC, SO, and HA prepared samples and performed angle dependent PL measurements. YC and HA fabricated devices. All authors discussed the results and commented on the manuscript.

\section{ACKNOWLEDGMENTS}

We gratefully acknowledge the partial financial support from the Center of Innovation (COI) Program from the Japan Science and Technology Agency, JST. HS acknowledges financial support in part by JSPS KAKENHI (17H03131) from JSPS.

\section{SUPPLEMENTARY MATERIAL}

The Supplementary Material for this article can be found online at: https://www.frontiersin.org/articles/10.3389/fchem. 2020.00427/full\#supplementary-material

Kim, K. H., and Kim, J. J. (2018). Origin and control of orientation of phosphorescent and TADF dyes for high-efficiency OLEDs. Adv. Mater. 30:1705600. doi: 10.1002/adma.201705600

Komatsu, R., Sasabe, H., Nakao, K., Hayasaka, Y., Ohsawa, T., and Kido, J. (2016a). Unlocking the potential of pyrimidine conjugate emitters to realize high-performance organic light-emitting devices. Adv. Opt. Mater. 5. doi: 10.1002/adom.201600675

Komatsu, R., Sasabe, H., Seino, Y., Nakao, K., and Kido, J. (2016b). Lightblue thermally activated delayed fluorescent emitters realizing a high external quantum efficiency of $25 \%$ and unprecedented low drive voltages in OLEDs. J. Mater. Chem. C 4:2274. doi: 10.1039/C5TC04057D

Komatsu, R.,Sasabe, H., and Kido, J. (2018). Recent progress of pyrimidine derivatives for high-performance organic light-emitting devices. J. Photon. Energy 8:032108. doi: 10.1117/1.JPE.8.032108

Komino, T., Sagara, Y., Tanaka, H., Oki, Y., Nakamura, N., Fujimoto, H., et al. (2016). Electroluminescence from completely horizontally oriented dye molecules. Appl. Phys. Lett. 108:241106. doi: 10.1063/1.4954163

Kondo, Y., Yoshiura, K., Kitera, S., Nishi, H., Oda, S., Gotoh, H., et al. (2019). Narrowband deep-blue organic light-emitting diode featuring an organoboronbased emitter. Nat. Photonics 13, 678-682. doi: 10.1038/s41566-0190476-5

Lee, Y. T., Tseng, P. C., Komino, T., Mamada, M., Ortiz, R. J., Leung, M. K., et al. (2018). Simple molecular-engineering approach for enhancing orientation and outcoupling efficiency of thermally activated delayed fluorescent emitters without red-shifting emission. ACS Appl. Mater. Interfaces 10, 43842-43849. doi: 10.1021/acsami.8b16199

Lin, M. S., Yang, S. J., Chang, H. W., Huang, Y. H., Tsai, Y. T., Wu, C. C., et al. (2012). Incorporation of a CN group into MCP: a new bipolar host material for highly efficient blue and white electrophosphorescent devices. J. Mater. Chem. 22, 16114-16120. doi: 10.1039/c2jm32717a

Lin, T. A., Chatterjee, T., Tsai, W. L., Lee, W. K., Wu, M. J., Jiao, M., et al. (2016). Sky-blue organic light emitting diode with $37 \%$ external quantum efficiency 
using thermally activated delayed fluorescence from spiroacridine-triazine hybrid. Adv. Mater. 28, 6976-6983. doi: 10.1002/adma.201601675

Liu, M., Komatsu, R., Cai, X., Hotta, K., Sato, S., Liu, K., et al. (2017). Horizontally orientated sticklike emitters: enhancement of intrinsic outcoupling factor and electroluminescence performance. Chem. Mater. 29, 8630-8636. doi: 10.1021/acs.chemmater.7b02403

Mayr, C., and Brütting, W. (2015). Control of molecular dye orientation in organic luminescent films by the glass transition temperature of the host material. Chem. Mater. 27, 2759-2762. doi: 10.1021/acs.chemmater.5b00062

Moon, C.-K., Kim, K.-H., Lee, J. W., and Kim, J.-J. (2015). Influence of host molecules on emitting dipole orientation of phosphorescent iridium complexes. Chem. Mater. 27, 2767-2769. doi: 10.1021/acs.chemmater.5b00469

Nakao, K., Sasabe, H., Komatsu, R., Hayasaka, Y., Ohsawa, T., and Kido, J. (2017). Significant enhancement of blue OLED performances through molecular engineering of pyrimidine-based emitter. Adv. Opt. Mater. 5:1600843. doi: 10.1002/adom.201600843

Nishio, M., Hirota, M., and Umezawa, Y. (1998). The CH/ $\pi$ Interaction-Evidence, Nature, and Consequences. New York, NY: Wiley-VCH.

Pal, A. K., Krotkus, S., Fontani, M., Mackenzie, C. F. R., Cordes, D. B., Slawin, A. M. Z., et al. (2018). High-efficiency deep-blue-emitting organic light-emitting diodes based on iridium(III) carbene complexes. Adv. Mater. 30:e1804231. doi: 10.1002/adma.201804231

Rajamalli, P., Senthilkumar, N., Huang, P. Y., Ren-Wu, C. C., Lin, H. W., and Cheng, C. H. (2017). New molecular design concurrently providing superior pure blue, thermally activated delayed fluorescence and optical outcoupling efficiencies. J. Am. Chem. Soc. 139, 10948-10951. doi: 10.1021/jacs. $7 \mathrm{~b} 03848$

Sasabe, H., Gonmori, E., Chiba, T., Li, Y. J., Tanaka, D., Su, S. J., et al. (2008). Wide-energy-gap electron-transport materials containing 3,5-dipyridylphenyl moieties for an ultra high efficiency blue organic light-emitting device. Chem. Mater. 20, 5951-5953. doi: 10.1021/cm801727d

Sasabe, H., and Kido, J. (2013). Development of high performance OLEDs for general lighting. J. Mater. Chem. C 1:1699. doi: 10.1039/c2tc00584k

Sasabe, H., Tanaka, D., Yokoyama, D., Chiba, T., Pu, Y.-J., Nakayama, K.-I., et al. (2011). Influence of substituted pyridine rings on physical properties and electron mobilities of 2-methylpyrimidine skeleton-based electron transporters. Adv. Func. Mater. 21, 336-342. doi: 10.1002/adfm.201001252

Schmidt, T. D., Lampe, T., Sylvinson, D. M. R., Djurovich, P. I., Thompson, M. E., and Brutting, W. (2017). Emitter orientation as a key parameter in organic light-emitting diodes. Phys. Rev. Appl. 8:037001. doi: 10.1103/PhysRevApplied.8.037001

Shibata, M., Sakai, Y., and Yokoyama, D. (2015). Advantages and disadvantages of vacuum-deposited and spin-coated amorphous organic semiconductor films for organic light-emitting diodes. J. Mater. Chem. C 3:11178. doi: 10.1039/C5TC01911G
Shin, H., Ha, Y. H., Kim, H. G., Kim, R., Kwon, S. K., Kim, Y. H., et al. (2019). Controlling horizontal dipole orientation and emission spectrum of Ir complexes by chemical design of ancillary ligands for efficient deep-blue organic light-emitting diodes. Adv. Mater. 31:1808102. doi: 10.1002/adma.201808102

Uoyama, H., Goushi, K., Shizu, K., Nomura, H., and Adachi, C. (2012). Highly efficient organic light-emitting diodes from delayed fluorescence. Nature 492, 234-238. doi: 10.1038/nature11687

Watanabe, Y., Sasabe, H., and Kido, J. (2019a). Review of molecular engineering for horizontal molecular orientation in organic light-emitting devices. Bull. Chem. Soc. Jpn. 92, 716-728. doi: 10.1246/bcsj.20180336

Watanabe, Y., Yokoyama, D., Koganezawa, T., Katagiri, H., Ito, T., Ohisa, S., et al. (2019b). Control of molecular orientation in organic semiconductor films using weak hydrogen bonds. Adv. Mater. 31:1808300. doi: 10.1002/adma.201808300

Wong, M. Y., and Zysman-Colman, E. (2017). Purely organic thermally activated delayed fluorescence materials for organic light-emitting diodes. Adv. Mater. 29:1605444. doi: 10.1002/adma.201605444

Wu, T.-L., Huang, M.-J., Lin, C.-C., Huang, P.-Y., Chou, T.-Y., Chen-Cheng, R.-W., et al. (2018). Diboron compound-based organic light-emitting diodes with high efficiency and reduced efficiency roll-off. Nat. Photonics 12, 235-240. doi: 10.1038/s41566-018-0112-9

Yang, Z. Y., Mao, Z., Xie, Z. L., Zhang, Y., Liu, S. W., Zhao, J., et al. (2017). Recent advances in organic thermally activated delayed fluorescence materials. Chem. Soc. Rev. 46, 915-1016. doi: 10.1039/C6CS00368K

Yokoyama, D. (2011). Molecular orientation in small-molecule organic lightemitting diodes. J. Mater. Chem. 21:19187. doi: 10.1039/c1jm13417e

Yokoyama, D., Sakaguchi, A., Suzuki, M., and Adachi, C. (2009). Horizontal orientation of linear-shaped organic molecules having bulky substituents in neat and doped vacuum-deposited amorphous films. Org. Electron. 10, 127-137. doi: 10.1016/j.orgel.2008.10.010

Yokoyama, D., Sasabe, H., Furukawa, Y., Adachi, C., and Kido, J. (2011). Molecular stacking induced by intermolecular $\mathrm{C}-\mathrm{H} \cdots \mathrm{N}$ hydrogen bonds leading to high carrier mobility in vacuum-deposited organic films. Adv. Func. Mater. 21, 1375-1382. doi: 10.1002/adfm.201001919

Conflict of Interest: The authors declare that the research was conducted in the absence of any commercial or financial relationships that could be construed as a potential conflict of interest.

Copyright (c) 2020 Sasabe, Chikayasu, Ohisa, Arai, Ohsawa, Komatsu, Watanabe, Yokoyama and Kido. This is an open-access article distributed under the terms of the Creative Commons Attribution License (CC BY). The use, distribution or reproduction in other forums is permitted, provided the original author $(s)$ and the copyright owner(s) are credited and that the original publication in this journal is cited, in accordance with accepted academic practice. No use, distribution or reproduction is permitted which does not comply with these terms. 